\title{
A propos de la filtration au-dessous des canaux
}

\section{Concerning seepage below canals}

\author{
par A. PREISSMANN
}

IABORATOIRES DE RECHERCHES HYDRAULIQUES FT DE MÉCANYQYE DES TERRES ANNEXÉs A L'ÉCOLE POLYTECHNIQUE FÉdÉRALE, ZURICH

\begin{abstract}
L'introduction définit les conditions de l'icoulement au-dessous d'un canal, et fait élat des méthodes utilisant lhodographe, en signalant leurs inconvénients.

L'autear propose une méthode générale donnant sans trop de calculs le réseau d'éconlement avec une exactitude suffisante. Principe de la méthode.

Applications. - Cas d'un canal de section triangulaire et symétrique. Cas des canaux de profil rectangulaire on trapézoïdal.

Extension de la méthode au cas où la profondeur a laquelle se tronve la conche tres perméable est faible.

Problème isopérimétrique: détermination de la forme de section permettant le débit d'infiltration minimum pour une aive imposés de la section.
\end{abstract}

\begin{abstract}
The Introduction defines the flow conditions below a canal and describes various means of utilising the hodograph, pointing out their disadvantages.

The author proposes a general method of arriving at the flow network with reasonable accuracy, without involved calculations. The method is then described.

Applications: The case of a canal of symmetrical, triangular section. The case of canals of rectangular or trapezoidal form.

Extension of the method to the case where the very permeable layer is not deep lying.

Isoperimetrical problem: determination of the section form giving the minimum seepage for a given section area.
\end{abstract}

\section{INTRODUCTION}

Lorsqu'un canal très long est creusé dans un sol perméable et que son lit n'est pas colmaté, une partie de l'eau s'écoule par infiltration audessous du canal. Le problème de la détermination de la perte d'eau et du réseau d'écoulement bimensionnel a déjà fait l'objet d'un certain nombre de travaux [1], [2], [3]. Il faut remarquer par ailleurs que les eonditions audessous du canal sont déterminantes pour l'écoulement. Ici nous supposerons que très loin audessous du canal se trouve une zone très perméable qui draine l'écoulement, ou autrement dit, qu'à une certaine distance du canal l'écoulement se rapproche d'un écoulement uniforme par gravité et que la pression se rapproche de la pression atmosphérique (fig. 1).

On admet que le sol est de perméabilité homogène et isotrope avec un coefficient de Darcy $k$. On sait que dans ce cas il existe un potenticl des vitesses :

$$
\varphi=k\left(y-\frac{p}{\gamma}\right)
$$

$p$ : pression, $\gamma$ : poids spécifique de l'eau. L'axe des $y$ est dirigé vers le bas.

$$
u=\frac{\partial \varphi}{\partial x}, v=\frac{\partial \varphi}{\partial y}
$$

$u, v:$ composante de la vitesse de filtration.

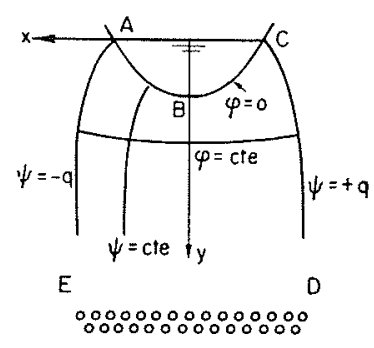

Fig. 1. - Schéma et désignations.

La fonction $q(x, y)$ est une fonction harmonique satisfaisant à l'équation $\Delta \varphi=0$, la fone- 
tion harmonique conjuguée $\psi(x, y)$ est dite fonction de courant. Les lignes $\psi=\mathrm{C}^{\text {te }}$ sont des li-

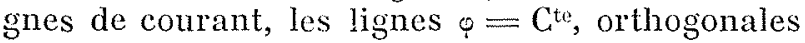
aux premières, sont dites lignes équipotentielles. La fonclion complexe :

$$
w=w(z)=\wp(x, y)+i \psi(x, y)
$$

est une fonction analytique de la variable complexe $x+i y$ qui détermine une représentation conforme de l'écoulement du plan des $(x, y)$ dans le plan des $w(\varphi, \psi)$.

La détermination de l'écoulement se réduit à un problème de la théorie du potentiel avec certaines conditions aux limites (voir fig. 1):

1. Sur le contour ABC (lit du canal), la pression est hydrostatique et l'on a $\varphi=0$.

2. Très loin au-dessous du canal, la pression s'annule : $\quad-k y \rightarrow 0$ pour $y \rightarrow \infty$.

3. Sur les surfaces libres $\mathrm{CD}$ et $\mathrm{AE}$, dont la forme $n$ 'est pas donnée à l'avance, on a deux conditions; d'une part, $\mathrm{CD}$ et $\mathrm{AE}$ sont des lignes de courant et donc $\psi=\mathrm{C}^{\text {te }}$, d'autre part, la pression s'annule sur ces lignes et l'on a $\varphi-k y=0$. Comme $\psi$ n'est donnée qu'à une constante près, on peut admettre $\psi=-q$ sur AE et $\psi=-q$ sur $C D, q$ représentant le demi-débit d'infiltration par unité de longueur du canal.

Ces conditions nous permettent immédiatement de délimiter le domaine du plan des $w$ correspondant au domaine d'infiltration dans le plan des $z$, et qui est un demi-ruban infini limité par les droites $\varphi=0$ et $\psi= \pm q$ (fig. 2).

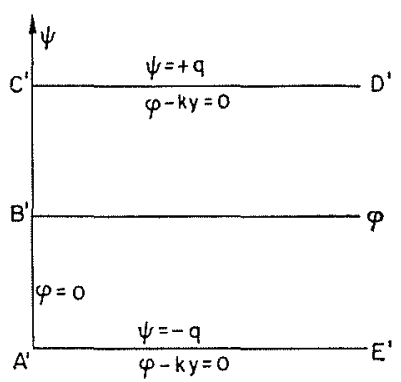

Fig. 2.

Représentation de l'écoulement sur le plan des $w$.

Les domaines $\mathrm{ABCDEA}$ et $\mathrm{A}^{\prime} \mathrm{B}^{\prime} \mathrm{C}^{\prime} \mathrm{D}^{\prime} \mathrm{E}^{\prime} \mathrm{A}^{\prime}$ sont reliés entre eux par la représentation conforme donnée par la fonction analytique $w(z)$. Or, la forme de ED et de EA n'est pas donnée à l'avance et doit être déterminée par la condition $\varphi-k y=0$.

Pour résoudre des problèmes de ce genre,
Helmholtz et KirchHoff ont introduit le plan de l'holographe (lieu des extrémités des vecteurs vitesses reportés à partir de l'origine des coordonnées). Dans notre cas, Vedernikov [3] s'est également servi de cette méthode; il a constaté que lorsque le profil du canal est polygonal, l'hodographe correspondant au contour de l'écoulement possède une forme donnée. Le problème de la détermination de l'écoulement souterrain est réduit ainsi à la représentation conforme du planl des $w$ (demi-ruban infini) sur le plan des $z$ (intérieur d'un triangle dans le cas du canal de profil triangulaire); ce dernier problème se résoud en principe à l'aide de la formule de Schwarz-Christoffel. La détermination de la représentation conforme du plan des $w$ sur le plan des $z$ exige encore une simple intégration.

La solution de VEDERnmov, si elle est parfaite du point de vue mathématique, exige un calcul numérique très considérable et ne s'applique qu'à des profils polygonaux. C'est pourquoi on a cherché des méthodes qui donnent sans trop de calculs le réseau d'écoulement avec une exactitude suffisante. Pavlovsiry [1] a développé une méthode de calcul approché qui se révèle être un cas particulier de la méthode que nous allons exposer (un seul terme considéré dans une série infinie).

\section{Principe de la méthode de calcul}

Nous savons que la fonction $w=0+i \psi$ est une fonction analytique de $z=x+i y$ qui transforme le domaine d'écoulement dans le demiruban infini $\varphi>0,|\psi|<q$.

Or, considérons la fonction inverse, également analytique, $z=z(w)$ et mettons-là sous la forme :

$$
z=\frac{i}{k}\left[w+z^{*}(w)\right]
$$

La fonction $z^{*}(w)$ est définie sur le demiruban infini $\varphi>0,|\psi|<q$. En vertu des conditions aux limites sur les deux surfaces libres $\downarrow= \pm q$, la partie réelle de $z^{*}(w)$ s'annule pour $\varphi>0, \psi= \pm q$. On peut alors, à l'aide du principe de symétrie de Schwarz, prolonger analytiquement la fonction $z^{*}(w)$ dans tout le demiplan $\varphi>0$ et on obtient ainsi une fonction périodique de période $4 q i$ satisfaisant également la relation de symétrie :

$$
z^{*}(i q+t)=\bar{z}^{*}(i q+\bar{t}) \quad(t \text { quelconque })
$$

Si l'on impose encore à la fonction $z^{*}(w)$ la condition de s'annuler lorsque $\varphi$ tend vers l'in- 
fini par valeurs positives, on constate que $z^{*}(w)$ se représente sous la forme:

$$
z^{*}(w)=\sum_{n=0}^{\infty} \mathrm{A}_{n} e^{-(2 n+1) \pi w / 2 \eta}-i \sum_{n=1}^{\infty} \mathrm{B}_{n} e^{-n \pi w / 4},
$$

où les coefficients $A_{n}$ et $B_{n}$ sont réels.

Si cette valeur de $z^{*}(w)$ est introduite dans l'équation (1), on trouve:

$$
l z z(w)=i w+i \sum_{n=0}^{\infty} \mathrm{A}_{n} e^{-(2 n+1) \pi w / 2 q}+\sum_{n=1}^{\infty} \mathrm{B}_{n} e^{-n \pi w / q}
$$

et, en séparant partie réelle et partie imaginaire :

$$
\left.\begin{array}{r}
k x=-\psi+\sum_{n=0}^{\infty} \mathrm{A}_{n} \sin \frac{(2 n+1) \pi \psi}{2 q} e^{-(2 n+1) \pi \varphi / 2 q} \\
+\sum_{n=1}^{\infty} \mathrm{B}_{n} \cos \frac{n \pi \psi}{q} e^{-n \pi \varphi / q} \\
k y=-\varphi+\sum_{n=0}^{\infty} \mathrm{A}_{n} \cos \frac{(2 n+1) \pi \psi}{2 q} e^{-(2 n+1) \pi \varphi / 2 q} \\
-\sum \mathrm{B}_{n} \sin \frac{n \pi \psi}{q} e^{-n \pi \varphi / q}
\end{array}\right\}
$$

formule donnant explicitement la représentation du plan $(\varphi, \downarrow)$ sur le plan $(x, y)$ si les coefficients $A_{n}$ et $B_{n}$ étaient connus.

On peut vérifier que pour $\psi= \pm q$, la deuxième des équations (3) nous donne $\varphi=k y$ et que, d'autre part, pour de grandes valeurs de ọ, l'écoulement tend bien vers un écoulement vertical uniforme : $k x=-\psi, k y=0$.

Nous avons done déjà tenu compte des conditions à la surface libre et trìs loin au-dessous du canal, il reste encore à considérer celle qui est relative au lit du canal : sur le lit du canal, le potentiel $\varphi$ des vitesses est nul.

Or, si l'on pose $\varphi=0$ dans les équations (3), on trouve :

$$
\begin{aligned}
& k x=-\psi+\sum_{n=0}^{\infty} A_{n} \sin \frac{2 n+1}{2 q} \pi \psi+\sum_{n=1}^{\infty} B_{n} \cos \frac{n \pi \psi}{q} \\
& k y=\quad \sum_{n=0}^{\infty} A_{n} \cos \frac{2 n+1}{2 q} \pi \psi-\sum_{n=1}^{\infty} B_{n} \sin \frac{n \pi \psi}{q}
\end{aligned}
$$

Ceci est une représentation paramétrique (paramètre $\psi$ ) de la ligne équipotentielle $\varphi=0 \mathrm{de}$ l'écoulement (3). La question qui reste à résou- dre est donc : Est-il possible de choisir les coefficients $A_{n}$ et $B_{n}$ de facon que la courbe définie par les équations (4) coïncide avec le profil du lit du canal?

Le problème dans sa généralité est difficile à résoudre. Toutefois, si l'on ne peut pas obtenir une solution exacte, mais seulement une solution suffisante pour les besoins de la pratique, on peut se limiter à un nombre fini $\mathrm{N}$ de termes des équations (4) et chercher la détermination des coefficients $\mathrm{A}_{n}$ et $\mathrm{B}_{n}$ de façon à approcher le mieux possible la forme du profil du canal. Pour un profil symétrique, par exemple, on se rend compte que tous les $\hat{\mathrm{B}}_{n 2}$ doivent être nuls et on peut écrire au lieu de (4):

$$
\begin{aligned}
& k x=-\psi+\sum_{n=0}^{N-1} \mathrm{~A}_{n} \sin \frac{(2 n+1) \pi \psi}{2 q} \\
& k y=\sum_{n=0}^{\mathrm{N}-1} A_{n} \cos \frac{(2 n+1) \pi \psi}{2 q}
\end{aligned}
$$

Soit $f(x, y)=0$ l'équation de la courbe effective du lit; comment choisir les $\mathrm{N}$ coefficients $A_{0}, A_{1}, \ldots, A_{x-1}$, de façon que la relation $f(x, y)=0$ soit satisfaite le mieux possible?

Si l'on remplace, dans l'expression $f(x, y)$, $x$ et $y$ par leur's valeur's tirées de $\left(4^{\prime}\right)$, on obtient une expression contenant $\psi$ et les coeficients $A_{0}, A_{1}, \ldots, A_{X-1}$; ce sont ces derniers cocfficients qui devront être choisis de façon que l'expression obtenue s'écarte le moins possible de zéro.

Le procédé, généralement employé dans ce but, consiste à considérer l'intégrale :

$$
\frac{1}{2 q} \int_{-\eta}^{q}[f(x(\psi), y(\psi))]^{2} d u
$$

qui représente l'erreur quadratique moyenne. Cette intégrale est une fonction des coefficients $A_{0}, \ldots, A_{x-1}$ (il a été intégré par rapport à $\psi$ ). En rendant cette erreur minimale par un choix judicieux des coefficients, on tire le meilleur parti possible de l'indétermination des formules (4). Il faut remarquer par ailleurs que le calcul des coefficients à partir de la condition du minimum n'est simple que dans le cas où la section est limitée par des droites; l'intégrale (5) est alors une forme quadratique des coefficients $A_{k}$ et la détermination des valeurs de ces coefficients est alors ramenée à la résolution d'un système d'équations linéaires.

Dans ce qui suit, nous donnerons quelques exemples d'applications de la méthode exposée ci-dessus. 


\section{Canal triangulaire}

Un canal de section triangulaire et symétrique est donné par la profondeur $t$ et l'angle $\alpha$ du remblai avec l'horizontale (fig. 3). Le canal est limité par les droites :

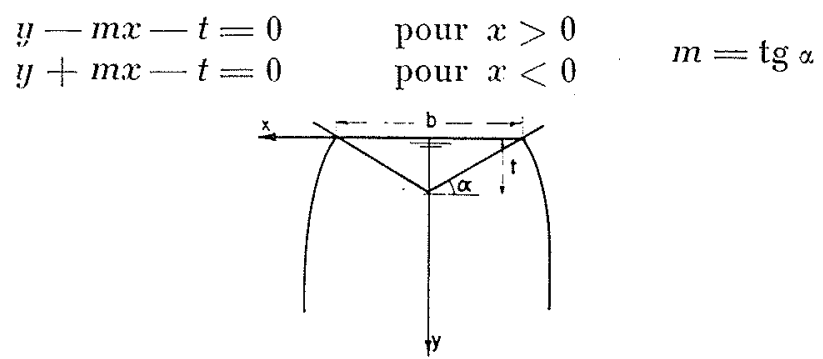

FIG. 3. - Profil triangulaire.

Comme le profil est symétrique, il suffira de ne considérer que la moitié de gauche $(x>0)$; en remplaçant $x$ et $y$ par leurs valeurs tirées de $\left(4^{\prime}\right) x(\psi)$ et $y(\psi)$, on est amené à considérer l'expression :

$$
\frac{1}{q} \int_{0}^{q}[y(\psi)-m x(\psi)-t]^{2} d \psi
$$

Le résultat de l'intégration est une forme quadratique en $A_{n}$ et $q$. En dérivant par rapport à ces variables on trouve un système de $\mathrm{N}+1$ équations linéaires permettant de trouver sans difficulté les valeurs cherchées des coefficients et du débit.

Pour des raisons de commodité, nous n'avons pas posé l'exigence $\left(5^{*}\right)$ mais une exigence analogue ayant trait à la meilleure approximation de la pente des berges, à savoir :

$$
\frac{1}{q} \int_{0}^{q}\left[\left(\frac{d y}{d \psi}\right)-m\left(\frac{d x}{d \psi}\right)\right]^{2} d \psi
$$

Si l'on n'a qu'un nombre très restreint de coefficients, il est clair que les résultats des deux méthodes peuvent être différents. Toutefois, la différence ne peut pas être importante si la condition (6) donne déjà une bonne approximation du profil choisi.

Si l'on remplace dans l'expression (6) les valeurs de $x$ et $y$ tirées de (4'), on trouve la forme quadratique :

$\frac{1}{k^{2}}\left\lceil\sum_{s} \mathrm{C}_{s}{ }^{2}\left\{\frac{1+m^{2}}{2}+\frac{2 m}{\pi(2 s+1)}\right\rceil+2 \sum_{\substack{s>t \\ s}} \mathrm{C}_{s} \mathrm{C}_{t} \frac{2 m}{\{+t+1}-\sum_{s} \mathrm{C}_{s} \frac{4 m}{\pi(2 s+1)}\left\{1+(-1)^{s} m\right\}+m^{2}\right]$

où :

$$
\mathrm{C}_{s}=\frac{2 s+1}{2 q} \pi \mathrm{A}_{s}
$$

$\mathrm{C}_{0}\left[1+m^{2}+\frac{4 m}{\pi}\right\rceil+\mathrm{C}_{2} \quad\left\lceil\frac{4 m}{3 \pi}\right\rceil+\mathrm{C}_{4} \quad\left\lceil\frac{4 m}{5 \pi}\right\rceil+\mathrm{C}_{\hat{0}} \quad\left\lceil\frac{4 m^{-}}{7 \pi}\right\rceil-\frac{4\left(m^{2}+m\right)}{\pi}=0$

$\mathrm{C}_{0} \quad\left\lceil\frac{4 m}{3 \pi}\right\rceil+\mathrm{C}_{2}\left\lceil 1+m^{2}+\frac{4 m}{5 \pi}\right\rceil+\mathrm{C}_{4} \quad\left\lceil\frac{4 m}{7 \pi}\right\rceil+\mathrm{C}_{6} \quad\left\lceil\frac{4 m}{9 \pi}\right\rceil \frac{4\left(m^{2}+m^{2}\right)}{5 \pi}=0$

$\mathrm{C}_{0} \quad\left\lceil\frac{4 m}{5 \pi}\right\rceil+\mathrm{C}_{2} \quad\left\lceil\frac{4 m}{7 \pi} \mid+\mathrm{C}_{4}\left\lceil 1+m^{2}+\frac{4 m}{9 \pi}\right\rfloor+\mathrm{C}_{0} \quad\left\lceil\frac{4 m}{11 \pi} \mid \frac{4\left(m^{2}+m\right)}{9 \pi}=0\right.\right.$

$\mathrm{C}_{9} \quad\left\lceil\frac{4 m}{7 \pi}\right\rceil+\mathrm{C}_{2} \quad\left[\frac{4 m}{9 \pi}\right\rceil+\mathrm{C}_{4} \quad\left\lceil\frac{4 m}{11 \pi}\right\rceil+\mathrm{C}_{66}\left\lceil 1+m^{2}+\frac{4 m}{13 \pi} \mid \frac{4\left(m^{2}+m\right)}{13 \pi}=0\right.$

$\mathrm{C}_{1}\left[1+m^{2}+\frac{4 m}{3 \pi}\left|+\mathrm{C}_{3} \quad\right| \frac{4 m}{5 \pi}\left|+\mathrm{C}_{5} \quad \quad\right| \frac{4 m}{7 \pi}\left|+\mathrm{C}_{7} \quad\right| \frac{4 m}{9 \pi} \mid \frac{4\left(m-m^{2}\right)}{3 \pi}=0\right.$

$\mathrm{C}_{1} \quad\left[\frac{4 m}{5 \pi}\right]+\mathrm{C}_{3} 1+m^{2}+\left\lceil\frac{4 m}{7 \pi}\right]+\mathrm{C}_{\overline{5}} \quad\left\lceil\frac{4 m}{9 \pi}\right]+\mathrm{C}_{\bar{\tau}} \quad\left\lceil\frac{4 m}{11 \pi}\right\rceil \frac{4\left(m-m^{2}\right)}{7 \pi}=0$

$\mathrm{C}_{1} \quad\left[\frac{4 m}{7 \pi}\right]+\mathrm{C}_{3} \quad\left[\frac{4 m}{9 \pi}\right]+\mathrm{C}_{5}\left[1+m^{2}+\frac{4 m}{11 \pi}\right]+\mathrm{C}_{\bar{\tau}} \quad\left[\frac{4 m}{13 \pi}\right]-\frac{4\left(m-m^{2}\right)}{11 \pi}=0$

$\mathrm{C}_{1} \quad\left[\frac{4 m}{9 \pi}\right]+\mathrm{C}_{3} \quad\left[\frac{4 m}{11 \pi}\right]+\mathrm{C}_{5} \quad\left[\frac{4 m}{13 \pi}\right]+\mathrm{C}_{\mathrm{i}}\left[1+m^{2}+\frac{4 m}{15 \pi}\right]-\frac{4\left(m-m^{2}\right)}{15 \pi}=0$ 
On voit que les valeurs de $C_{k}$, qui rendent la forme quadratique (7) minimale, ne dépendent pas de $q$.

Les conditions pour que la forme (7) soit minimum s'obtiennent par dérivation par rapport aux coefficients $A_{*}$. Nous écrivons ces conditions lorsque, comme nous l'avons fait pour le calcul numérique, on n'a gardé que 8 coefficients $A_{n}$.

La loi de formation des coefficients est immédiatement visible. Le système de 8 équations a 8 inconnues se décompose ici en deux systèmes de 4 équations et 4 inconnues. Le calcul effectué montre qu'avec seulement 8 termes on obtient une très bonne approximation du profil prescrit.

Dans le cas particulier $m=1$ (remblais à $45^{\circ}$ ), on voit immédiatement que :

$$
\mathrm{C}_{1}=\mathrm{C}_{3}=\mathrm{C}_{5}=\mathrm{C}_{\tau}=0
$$

parce que le terme connu dans les quatre dernières équations (8) est nul.

Les valeurs de $C_{0}, C_{2}, C_{4}, C_{6}$ et celles correspondantes de $A_{0}, A_{2}, A_{4}, A_{6}$ sont :

$$
\begin{array}{ll}
\mathrm{C}_{0}=0,762 & \mathrm{~A}_{0}=0,485 q \\
\mathrm{C}_{2}=0,097 & \mathrm{~A}_{2}=0,0123 q \\
\mathrm{C}_{4}=0,032 & \mathrm{~A}_{4}=0,0023 q \\
\mathrm{C}_{0}=0,019 & \mathrm{~A}_{6}=0,0009 q
\end{array}
$$

On obtient les équations du mouvement en remplaçant dans les équations (3) les coefficients $A_{n}$ par leur valeur (les $B_{n}$ sont nuls, ainsi que les $A_{n}$ d'indice impair).

Ce mouvement de filtration correspond au profil donné par la représentation paramétrique :

$$
\begin{aligned}
\frac{k x}{q}= & \frac{\psi}{q}+0,485 \sin \frac{\pi \psi}{2 q}+0,0123 \sin \frac{3 \pi \psi}{2 q} \\
& +0,0023 \sin \frac{5 \pi \psi}{2 q}+0,0009 \sin \frac{7 \pi \psi}{2 q} \\
\frac{k y}{q}=\quad & +0,495 \cos \frac{\pi \psi}{2 q}+0,0123 \cos \frac{3 \pi \psi}{2 q} \\
& +0,0023 \sin \frac{5 \pi \psi}{2 q}+0,0009 \sin \frac{7 \pi \psi}{2 q}
\end{aligned}
$$

On peut dessiner ce profil et l'on constate qu'il diffère très peu du profil triangulaire choisi; pour $x=0$, on a $\psi=0$ et $y=0,5 q$.

Dans la réalité, ce n'est pas q qui est donné, mais par exemple la profondeur $t$ du canal. Le calcul s'exécute alors comme suit : on détermine d'abord les $\mathrm{C}_{*}$ qui ne dépendent pas de $q$.
Les valeurs de $A_{s}$ sont proportionnelles à $q$, la valeur de $k y / q$ pour $x=0$, c'est-à-dire l'expression $k t / q$, est indépendante de $q$. Lorsque $t$ est donnée, on peut calculer ainsi la valeur de $q$. Dans notre cas $(m=1)$, on trouve $q=2 \mathrm{kt}$. La quantité d'ean perdue par infiltration est le double de celle qui correspond à un canal très plat de même largeur. Pour $m=(1 / 2)$, les calculs conduisent aux valeurs :

$$
\begin{array}{ll}
A_{0}=0,316 q & A_{4}=0,0020 q \\
A_{1}=0,0146 q & A_{5}=0,0009 q \\
A_{2}=0,0076 q & A_{6}=0,0009 q \\
A_{3}=0,0023 q & A_{5}=0,0005 q
\end{array}
$$

Ici également, la ligne équipotentielle $\circ=0$ est très voisine du contour triangulaire donné. La profondeur est égale à $0,345(q / k)$. Ainsi on a $q=(k t / 0,345)$. La quantité d'eau perdue par infiltration est 1,45 fois celle qui correspond à un canal très plat de même largeur.

Pour $m=2$, on trouve :

$$
\begin{array}{ll}
A_{0}=0,632 q & A_{i}=0,0040 q \\
A_{1}=-0,0292 q & A_{5}=-0,0019 q \\
A_{2}=0,0151 q & A_{6}=0,0018 q \\
A_{3}=-0,0046 q & A_{i}=-0,0010 q
\end{array}
$$

Ici la convergence de l'écuipotentielle $\bullet=0$ vers la forme prescrite du canal est moins bonne. Remarquons que les coefficients $\mathrm{A}_{s}$ pow $m=2$ se déduisent des valeurs $\mathrm{A}_{s}$ correspondant à $m=(1 / 2)$ de la facon suivante : il suffit de multiplier par 2 les coefficients d'ordre pair et par - 2 les coefficients d'ordre impair. Cefte remarque peut se généraliser : on trouve les coefficients pour la pente $1 / m$ à partir des coefficients pour la pente $m$ en multipliant par $1 / m$ les coefficients d'ordre pair of par $-1 / \mathrm{m}$ Jes coefficients d'ordre impair.

Pour $m=2$, la profondeur est égale à $0,610(q / k)$ et la largeur à $2 \times 0,310(q / k)$ (i) $\mathrm{y}$ a ici une erreur due an nombre restreint des termes). La quantité perduc par infiltration est ćgale à 3,2 fois celle oui correspond à un canal très peu profond de même largeur.

Ces exemples confirment la relation approchée de Venernikov [3]:

$$
2 q / k=b+\lceil 2,123(x / \pi)+1,47\rceil t
$$

où $b$ représente la largeur totale du canal, $t$ ln profondeur, et a est l'angle des remblais avec I'horizontale. 


\section{Canal rectangulaire}

Dans le cas d'un profil rectangulaire (fig. 4) il s'agit d'exprimer que la coordonnée :

$$
y=\Sigma \mathrm{A}_{n} \cos \frac{2 n+1}{2 q} \pi \psi
$$

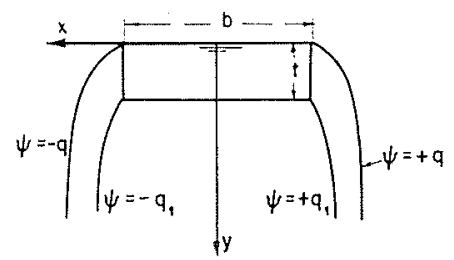

Fig. 4. - Profil rectangulaire.

reste constante égale à $t$ pour $\psi$ compris entre 0 et une certaine valeur $\varphi_{1}$ et que d'autre part la coordonnée :

$$
x=-\frac{\psi}{k}+\Sigma A_{n} \sin \frac{2 n+1}{2 q} \pi \downarrow
$$

reste constante, égale à $-b$ lorsque $\psi$ varie de $q_{1}$ à $q$. On ne connaît à l'avance ni la valeur de $q$, ni la valeur u. du rapport $q_{1} / q$.

Pour déterminer les valeurs des coefficients et les débits $q_{1}$ et $q$, on exprime que la somme des intégrales :

$$
\begin{aligned}
\frac{1}{b} & {\left[\int_{0}^{q_{1}}\left(\sum_{1}^{x-1} A_{n} \cos \frac{2 n+1}{2 q} \pi \psi-k t\right)^{2} d \psi+\right.} \\
& \left.\int_{0}^{q}\left(-\psi+\sum_{I}^{N-1} A_{n} \sin \frac{2 n+1}{2 q} \pi \psi+k b\right)^{2} d \psi\right]
\end{aligned}
$$

est un minimum.

Lorsqu'on effectue les calculs, on voit que les intégrales sont des formes quadratiqües en $A_{n}$ et en $q$ dont les coefficients sont des fonctions trigonométriques du rapport $\varphi_{0}=q_{1} / q$. En exprimant les conditions du minimum, on arrive à $\mathrm{N}+1$ équations linéaires en $\mathrm{A}_{n}$ et $q$ et une équation transcendante en $y$. et quadratique en $\mathrm{A}_{n}$.

Nous avons procédé ici de façon quelque peu différente. Comme dans le cas du profil triangulaire, on a cherché à approcher la tangente au profil en rendant minimale l'expression :

$$
\frac{1}{q}\left[\int_{0}^{q_{1}}\left(\frac{d y}{d \psi}\right)^{2} d \psi+\int_{q 1}^{q}\left(\frac{d x}{d \psi}\right)^{2} d \psi\right]
$$

Tous calculs effectués, l'expression (9) se met sous la forme:

$$
\begin{aligned}
& \frac{1}{\pi k^{2}}\left\{\sum_{s} \mathrm{C}_{s}{ }^{2} \mid \frac{\pi^{\prime}}{2}-\frac{1}{2 s+1} \sin (2 s+1) \pi u\right] \\
& -2 \sum_{s>t} \mathrm{C}_{s} \mathrm{C}_{t} \frac{1}{s+t+1} \sin (s+t+1) \pi u \\
& -2 \sum_{s} \mathrm{C}_{s} \frac{2}{2 s+1}\left[(-1)^{5}-\sin \frac{2 s+1}{2} \pi u\right]+1-\psi \\
& y=q_{1} / q \quad \mathrm{C}_{s}=\frac{2 s+1}{2 q} \pi A_{s}
\end{aligned}
$$

La valeur de $q$ ne figure pas explicitement dans l'expression (10) Au lieu de donner $b$ ct $t$ et de calculer les valeurs de $\mu$ et de $q$, nous avons ici admis des valeurs de $\mu=q_{1} / q$ et dans chaque cas on peut calculer le rapport $t / b$. Le calcul a été effectué pour les valeurs de v. : $0,1,0,2,0,3,0,5,0,7,0,8$ et 0,9 avec 8 coefficients. Les résultats permettent de construire $k t / q$ et $k b / q$ en fonction de $\mu$ (fig. 5). Inverse-

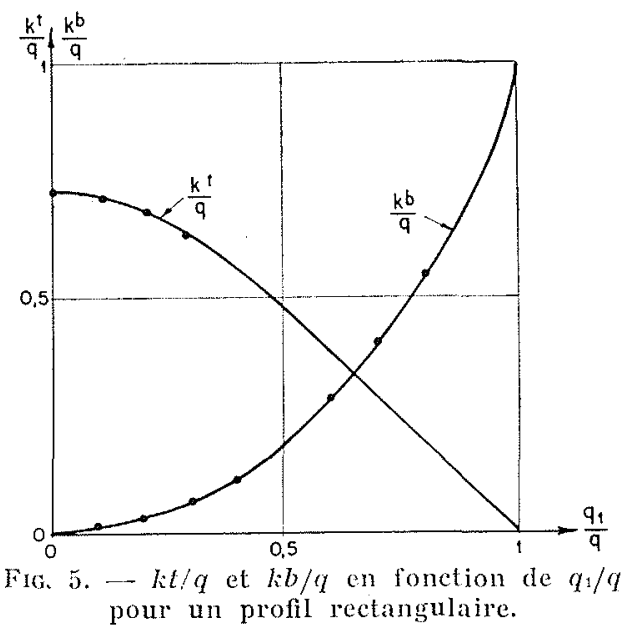

ment, ces diagrammes permettent de déterminer $q$ et $q_{1}$ pour un profil rectangulaire donné.

Dans le cas du profil rectangulaire, la convergence est moins bonne que dans le cas du profil triangulaire.

\section{Profil trapézoïdal}

Le cas du trapèze n'offre pas de difficulté de principe (fig. 6).

Ici encore, il faut exprimer que sur l'équipotentielle $\varphi=0$ :

$$
\begin{array}{lll}
\frac{d y}{d \Psi}=0 & \text { pour } & 0<\psi<q_{1} \\
\frac{d y}{d \Psi}-m \frac{d x}{d \Psi}=0 & \text { pour } & q_{1}<\psi<q
\end{array}
$$


Encore une fois, on écrit $x$ et $y$ sous la forme $\left(4^{\prime}\right)$ et on rend minimale l'expression :

$\frac{1}{q}\left[\int_{0}^{q_{1}}\left(\frac{d y}{d \psi}\right)^{2} d \psi+\int_{q_{1}}^{q_{1}}\left(\frac{d y}{d \psi}-m \frac{d x}{d \psi}\right)^{2} d \psi\right]$

Le calcul s'effectue de la même façon que dans le cas du profil rectangulaire. A une valeur

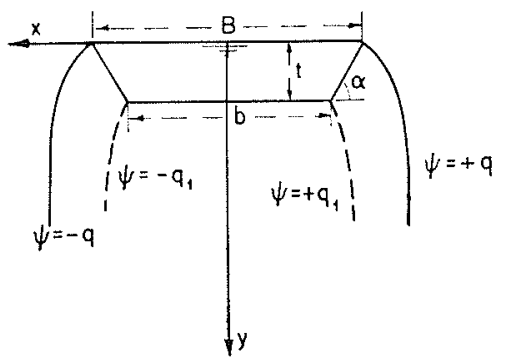

Fic. 6. - Profil trapézoïdal.

particulière du raport $q_{1} / q$ correspondra une valeur déterminée du quotient hauteur sur largeur moyenne.

Les calculs effectués dans le cas :

$$
m=1\left(\alpha=45^{\circ}\right) \text { et } q_{1} / q=1 / 2
$$

domnent avec 4 termes :

$$
\mathrm{B}=0,59 \frac{q}{k}, \quad b=0,30 \frac{q}{k}, \quad t=0,29 \frac{q}{k}
$$

\section{Extension de la méthode}

Les exemples précédents supposent que la couche très perméable se trouve très loin audessous du canal. Cette hypothèse n'est pas nécessaire. Soit $y_{0}$ la profondeur de la couche très perméable au-dessous du niveau de la surface libre du canal, on peut vérifier que les expressions :
La courbe équipotentielle $\varphi=0$ possède la représentation paramétrique suivante :

$k x=--\psi+\sum_{n=0}^{\infty} A_{n} \operatorname{cth} \frac{2 n+1}{2 q} \pi k y_{0} \sin \frac{2 n+1}{2 q} \pi \psi$

$k y=\quad \sum_{n=0}^{\infty} A_{n} \cos \frac{2 n+1}{2 q} \pi \psi$

Il s'agira ici aussi de déterminer les coefficients $A_{n}$ de telle façon que la courbe (14) soit identique au profil donné symétrique (le cas asymétrique se résoud de façon analogue). Le procédé est exactement analogue à celui du cas $y_{0}=\infty$, la seule différence résidant dans la présence du facteur Cth $(2 n+1 / 2 q) \pi k y_{0}$. Or, le quotient $k y_{0} / 2 q$ représente le rapport entre la profondeur $y_{0}$ et la largeur d'un écoulement uniforme vertical de même débit. Si ce rapport est égal à 1 , la valeur de $\operatorname{Cth}(2 n+1 / 2 q) \pi k y_{0}$ est 1,00037 pour $n=0$ et pratiquement égal à 1 pour $n>1$. Ainsi, dans ce cas, comme dans beaucoup d'autres cas pratiques, on peut appliquer sans erreur appréciable la méthode développée dans les paragraphes précédents, même si la profondeur n'est pas très grande.

\section{Un problème isopérimétrique}

On peut se poser lai question suivante :

Parmi toutes les formes possibles de la section du canal, quelle est celle qui donne le débit d'infiltration minimum lorsque l'aire de la section du canal est prescrite?

Ce problème qui, à première vue, paraît très difficile, est en réalité très simple si l'on applique la méthode développée dans les paragraphes précédents. En effet, le problème énoncé peut également se formuler :

Parmi toutes les formes de canal qui donnent

$$
\left.\begin{array}{l}
k x=-\psi+\sum_{n=0}^{\infty} \mathrm{A}_{n} \sin \frac{2 n+1}{2 q} \pi \psi \frac{\operatorname{Ch}(2 n+1 / 2 q) \pi\left(k y_{0}-0\right)}{\operatorname{Sh}(2 n+1 / 2 q) \pi k y_{0}} \\
k y=-\varphi+\sum_{n=0}^{\infty} A_{n} \cos \frac{2 n+1}{2 q} \pi \psi \frac{\operatorname{Sh}(2 n+1 / 2 q) \pi\left(k y_{0}-\varphi\right)}{\operatorname{Sh}(2 n+1 / 2 q) \pi k y_{0}}
\end{array}\right\}
$$

satisfont aux conditions d'un écoulement potentiel et aux conditions aux limites sur la surface libre et sur le plan $y=y_{0}$. En effet, on a :

$$
\begin{aligned}
\frac{\partial x}{\partial \varphi} & =\frac{\partial y}{\partial \psi}, & \frac{\partial x}{\partial \psi}=-\frac{\partial y}{\partial \varphi} \\
\text { pour } \psi= \pm q: & & k y-\varphi=0 \\
\text { pour } \varphi=k y: & & y=y_{0}
\end{aligned}
$$

le même débit d'infiltration, quelle est celle qui possède la plus grande aire

Si $q$ est donnée, on peut écrire l'équation paramétrique de la forme du canal sous la forme (4') et calculer l'aire de la section du canal en fonction des coefficients $\mathrm{A}_{n}$ :

$$
\mathrm{F}=\int_{-q}^{+q} y(\psi) \frac{d x(\psi)}{d \psi} d \psi
$$


Le calcul de l'intégrale donne:

$\mathrm{F}=\frac{2}{k^{2}} \frac{2}{\pi}\left(\sum_{n=1}^{\infty}(-1)^{n} \frac{\mathrm{A}_{n}}{2 n+1}-\frac{\pi}{4} \sum_{n=11}^{\infty}(2 n+1) \mathrm{A}_{n^{2}}{ }^{2}\right.$

$\left.-\frac{\pi}{2} \sum_{n=1}^{\infty} n B_{n}{ }^{2}\right)=\frac{2}{k^{2}} \frac{4 q^{2}}{\pi^{3}} \mid \sum_{n=0}^{\infty} \frac{1}{(2 n+1)^{3}}-\frac{\pi}{4}$

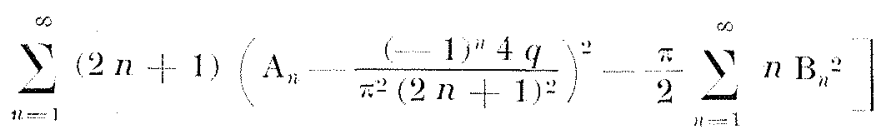

Tous les termes des deux dernières sommes étant positifs ou nuls, on aura :

$$
\mathrm{F} \leqslant \frac{8 q^{2}}{k^{2}} \frac{1}{\pi^{3}} \sum_{n=0}^{\infty} \frac{1}{(2 n+1)^{3}} \sim 0,282 \frac{q^{2}}{k^{2}}
$$

L'égalité n'a lieu que lorsque $B_{n}=0$ el $A_{n}=(-1)^{n} 4 q / \pi^{2}(2 n+1)^{2}$ pour toutes les valeurs de $n$. On peut aussi ćcrire l'équation (15) sous la forme:

$$
q^{2} \geqslant \frac{k^{2} \pi^{3} \mathrm{~F}}{8 \geq(1 / 2 n+1)^{3}} \sim 3,55 \mathrm{~F} k^{2}
$$

Ainsi le problème isopérimétrique est résolu si l'on admet à concurrence tous les profils qui peuvent se mettre sous la forme (4).

Examinons encore le profil symétrique qui donne le minimum d'infiltration et qu'on obtient en posant $\mathrm{B}_{n}=0$ et $\mathrm{A}_{n}=(-1)^{n} 4 q / \pi^{2}(2 n+1)^{2}$ dans les équations (4'). On trouve:

$$
\begin{aligned}
& k x=-\psi+\frac{4 q}{\pi^{2}} \sum_{n=0}^{\infty} \frac{(-1)^{n}}{(2 n+1)^{2}} \sin \frac{2 n+1}{2 q} \pi \psi \\
& k y=\quad \frac{4 q}{\pi^{2}} \sum_{n=0}^{\infty} \frac{(-1)^{n}}{(2 n+1)^{2}} \cos \frac{2 n+1}{2 q} \pi \psi
\end{aligned}
$$

Or, dans l'intervalle considéré $-q<\psi<+q$, l'expression :

$$
\frac{4 q}{\pi^{2}} \sum_{n=0}^{\infty} \frac{(-1)^{n}}{(2 n+1)^{2}} \sin \frac{2 n+1}{2 q} \pi \psi
$$

est simplement égale à $\downarrow / 2$, de sorte que :

$$
k x=-\frac{\psi}{2}
$$

Ainsi done, sur le profil considéré, le débit d'infiltration entre deux points du lit est simplement proportionnel à la différence des abscisses de ces deux points. Cette propriété permet, par utilisation de la représentation con-

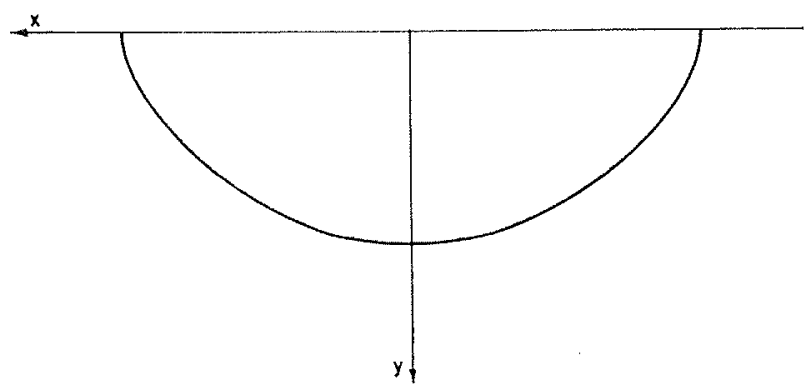

Fig. 7. - Profil donnant le débit d'infiltration minimum pour une aire donnée.

forme du plan des $w$ et des $d z / d w$ (plans utilisés par Vedernikov) de donner une expression analytique pour la forme du contour. Tous calculs faits, l'équation du profil s'écrit :

$$
y=\frac{1}{\pi} \int_{-q / 2 k}^{x} \ln \left(\frac{1-\sin \pi k x / q}{1+\sin \pi k x / q}\right) d x
$$

Ce profil est représenté sur la figure 7 .

\section{BIBLIOGRAPHIE}

[1] Pavlovsin (N.M.) : Bulletin de l'Institut de recherches hydrotechniques. Leningrad, lome 19, 1936 , pp. 1-24, 49-81.

[2] Vedennikov (V.) : Wasserkraft und Wasserwirtschaft 1936 , p. 128

[3] VenenNiov (V.): Zeitschrift für angemandte Mathematik und Mechanil, vol. 17, 1937, p. 155 . 